\title{
Competências do enfermeiro na promoção da saúde da pessoa idosa com hipertensão arterial sistêmica
}

\author{
Nurses' competences in promoting the health of elderly people with systemic arterial hypertension
}

Habilidades del enfermero en la promoción de la salud de los ancianos con hipertensión arterial sistémica

\author{
Francisco Wellington Dourado Júnior' ${ }^{\circledR}$; Jamylle Lucas Diniz"l ${ }^{\circledR}$; Andréa Carvalho Araújo Moreiral $\odot$; \\ Dafne Lopes Salles' ${ }^{\circledR}$; Luís Henrique Azevedo Moreira' ${ }^{\circledR}$
}

'Universidade Estadual Vale do Acaraú, Sobral, CE, Brasil; "Universidade Federal do Ceará, Fortaleza, CE, Brasil

\begin{abstract}
RESUMO
Objetivo: identificar na literatura as competências do enfermeiro relacionadas à promoção da saúde de idosos com hipertensão arterial sistêmica (HAS) à luz do Consenso de Galway. Método: trata-se de uma revisão integrativa da literatura, cuja busca foi realizada nas bases de dados LILACS, MEDLINE/PubMed, BDENF, SCOPUS e COCHRANE, de artigos que datassem de 2011 à 2020. Dos 11.892 artigos encontrados, 20 foram selecionados. Resultados: os artigos foram analisados de acordo com os oito domínios do modelo de competências para promoção da saúde de Galway: catalisar mudanças, liderança, avaliação das necessidades, planejamento, implementação, avaliação do impacto, parceria e defesa. Os domínios mais evidenciados foram catalisar mudanças, avaliação do impacto e planejamento. Conclusão: os resultados desta pesquisa evidenciaram o protagonismo do enfermeiro na promoção da saúde de idosos hipertensos, capaz de atuar na gestão do cuidado qualificado e contribuir com a assistência integral à pessoa idosa.
\end{abstract}

Descritores: Enfermagem; Competência Profissional; Promoção da Saúde; Idoso; Doença Crônica.

\begin{abstract}
Objective: to identify, in the literature, nurses' competences related to promoting the health of elderly people with systemic arterial hypertension (SAH) in light of the Galway Consensus. Method: in this integrative literature review, the LILACS, MEDLINE/PubMed, BDENF, SCOPUS, and COCHRANE databases were searched for articles dating from 2011 to 2020. Of the 11,892 articles found, 20 were selected. Results: the articles were analyzed by the eight domains of the Galway model of health promotion competences: catalyzing change, leadership, needs assessment, planning, implementation, impact assessment, partnership, and advocacy. The domains most in evidence were catalyzing change, impact assessment, and planning. Conclusion: the results of this research showed that nurses play a leading role in promoting the health of hypertensive elderly people and are capable of acting in management of skilled care and of contributing to comprehensive care for the elderly. Descriptors: Nursing; Professional Competence; Health Promotion; Aged; Chronic Disease.

\section{RESUMEN}

Objetivo: identificar en la literatura las habilidades del enfermero relacionadas a la promoción de salud de los ancianos con hipertensión arterial sistémica (HAS) de acuerdo con el Consenso de Galway. Método: es una revisión integradora de la literatura, cuya búsqueda se ha realizado en las bases de datos LILACS, MEDLINE/PubMed, BDENF, SCOPUS y COCHRANE, de artículos publicados entre 2011 y 2020. De los 11.892 artículos encontrados, 20 han sido seleccionados. Resultados: los artículos han sido analizados de acuerdo con los ocho dominios del modelo de habilidades para la promoción de la salud de Galway: catalizar cambios, liderazgo, evaluación de las necesidades, planificación, implementación, evaluación del impacto, colaboración y defensa. Los dominios con más evidencia han sido: catalizar cambios, evaluar el impacto y la planificación. Conclusión: los resultados de esta investigación han comprobado el protagonismo del enfermero en la promoción de la salud de ancianos hipertensos, capaz de actuar en la gestión del cuidado cualificado y contribuir con la asistencia integral al anciano. Descriptores: Enfermería; Competencia Profesional; Promoción de la Salud; Anciano; Enfermedad Crónica.
\end{abstract}

\section{INTRODUÇÃO}

As doenças crônicas não transmissíveis (DCNT) são as principais causas de mortalidade e incapacidade em todo o mundo. Anualmente, cerca de 38 milhões de óbitos são acarretados por essas doenças, sendo que $75 \%$ desse total são registrados em países de baixa e média renda como o Brasil ${ }^{1}$.

Dentre as DCNT, a mais comum em pessoas idosas é a Hipertensão Arterial Sistêmica (HAS) que pode interferir na realização das atividades de vida diária, bem como acarretar repercussões significativas na vida do paciente, como mudanças no cotidiano do idoso e de seus familiares frente a adoção de rotinas e comportamentos relacionados a práticas mais saudáveis². Nesse contexto, a adoção de medidas para promoção da saúde é fundamental para o envelhecimento saudável, na medida que atenuam o risco de fragilização e incapacidades funcionais ${ }^{3}$.

Autor correspondente: Francisco Wellington Dourado Júnior. E-mail: wjunior0599@gmail.com

Editora Científica: Cristiane Helena Gallasch; Editor Associado: Antonio Marcos Tosoli Gomes 
Desse modo, surge a necessidade da atuação de enfermeiros na assistência integral a pessoa idosa, não apenas em uma dimensão curativa, mas com enfoque na prevenção de comorbidades e na realização de atividades de promoção da saúde, baseadas em estratégias que englobem todo o contexto biopsicossocial do idoso e da sua família, a fim de suprir necessidades primárias ${ }^{4}$.

Nesse sentido, em junho de 2008 foi pactuado o Consenso de Galway em que subsidia a implementação das ações de promoção da saúde por meio da padronização de competências fundamentais, incentivando a adoção de práticas de qualidade pelos profissionais de saúde, uma vez que são incentivadas novas atitudes no processo de trabalho ${ }^{4}$. 0 documento ainda ressalta os valores e princípios de promoção da saúde baseados nos determinantes de saúde, na equidade, justiça social e respeito às diversidades, bem como determina as competências essenciais para promover saúde, divididas nos seguintes domínios: Catalisar mudança, Liderança, Avaliação das necessidades, Planejamento, Implementação, Avaliação, Advocacia e Parcerias ${ }^{5}$.

Dessa forma, identificar intervenções de enfermagem realizadas para promoção de saúde dos idosos hipertensos de acordo com Consenso de Galway corrobora para o desenvolvimento da prática de enfermagem baseada em evidências e subsidia uma assistência de qualidade, com enfoque nas necessidades do sujeito e não mais centralizada na doença. Além disso, espera-se que o presente estudo promova uma reflexão crítica sobre a atuação do enfermeiro no contexto da promoção da saúde de pessoas idosas com hipertensão, a fim de fortalecer a prática profissional na execução do cuidado integral.

Nessa perspectiva, este estudo teve por objetivo identificar na literatura as competências do enfermeiro relacionadas à promoção da saúde de idosos com hipertensão arterial sistêmica (HAS) à luz do Consenso de Galway.

\section{MÉTODO}

Trata-se de revisão integrativa realizada em seis etapas, a saber: elaboração da questão norteadora; definição das bases de dados e dos critérios de inclusão e exclusão da amostra; definição das informações a serem extraídas dos estudos selecionados; avaliação dos estudos incluídos na revisão integrativa; interpretação dos resultados e, por último, apresentação da revisão/síntese do conhecimento produzido ${ }^{6}$.

Para formação da pergunta norteadora foi utilizada a estratégia PICo (População /paciente: idosos hipertensos; IIntervenção: intervenção de enfermagem; Co-Contexto: promoção da saúde de acordo com o consenso de Galway), o que resultou na seguinte questão de pesquisa: Quais intervenções de enfermagem são realizadas para promoção da saúde de idosos hipertensos de acordo com o consenso de Galway? ${ }^{7}$.

Os artigos foram identificados através de uma busca bibliográfica realizada no mês de abril de 2020 por dois pesquisadores independentes nas seguintes bases de dados: LiteraturaLatino-Americana em Ciências de Saúde (LILACS) e Base de Dados em Enfermagem (BDENF), consultadas pela Biblioteca Virtual de Saúde (BVS), Medical Literature Analysis and Retrieval System Online (MEDLINE), via PubMed, SCOPUS e Cochrane acessadas pelo Portal CAPES.

Os critérios para inclusão adotados foram: artigos disponíveis eletronicamente, nos idiomas inglês, espanhol e/ou português, publicados no período de janeiro de 2011 a março de 2020, cuja pesquisa tratasse de pessoas de 60 anos ou mais com hipertensão arterial sistêmica e que versassem sobre intervenções de Enfermagem para promoção de saúde. A delimitação do tempo do estudo teve como marco a Política Nacional de Promoção da Saúde, a qual ressalta a necessidade de práticas de controle dos determinantes de saúde ${ }^{8}$. Foram excluídos os editoriais, cartas ao editor, trabalhos publicados em anais de evento, artigos de reflexão e artigos repetidos.

A realização das buscas de dados nas bases internacionais utilizou os descritores Medical Subject Headings (MeSH) com os respectivos operadores booleanos: Hypertension AND Aged OR Aging AND Nursing Care OR Nusing Process. Nas buscas da BVS foram utilizados os Descritores em Ciências da Saúde (DeCS) também combinados com operadores boleanos, sendo eles Hipertensão AND Idosos AND Cuidados de Enfermagem OR Processo de Enfermagem.

Encontrou-se um total de 11.892 publicações. Após a identificação dos estudos, foi realizado leitura dos títulos e resumos, sendo excluídos todos aqueles que não atendiam aos critérios de inclusão e/ou ao tema proposto. Depois deu-se início à leitura na íntegra dos estudos, no qual foram excluídos os estudos duplicados. Por fim, foram selecionados 20 artigos para compor amostra final da revisão. Para descrição das buscas e seleção dos estudos utilizouse o Preferred Reporting Items for Systematic Review and Meta-Analyses (PRISMA) ${ }^{9}$.

A qualidade dos estudos foi avaliada com base na classificação do nível de evidência, a saber: nível I - evidência obtida do resultado de metanálise de estudos clínicos controlados e com randomização; nível II- evidência obtida em estudo de desenho experimental; nível III - evidência obtida de pesquisas quase-experimentais; nível IV - evidências obtidas de estudos descritivos ou com abordagem metodológica qualitativa; nível V - evidências obtidas de relatórios de casos ou relatos de experiências; nível $\mathrm{VI}$ - evidências baseadas em opiniões de especialistas ou com base em normas ou legislação ${ }^{6}$. 


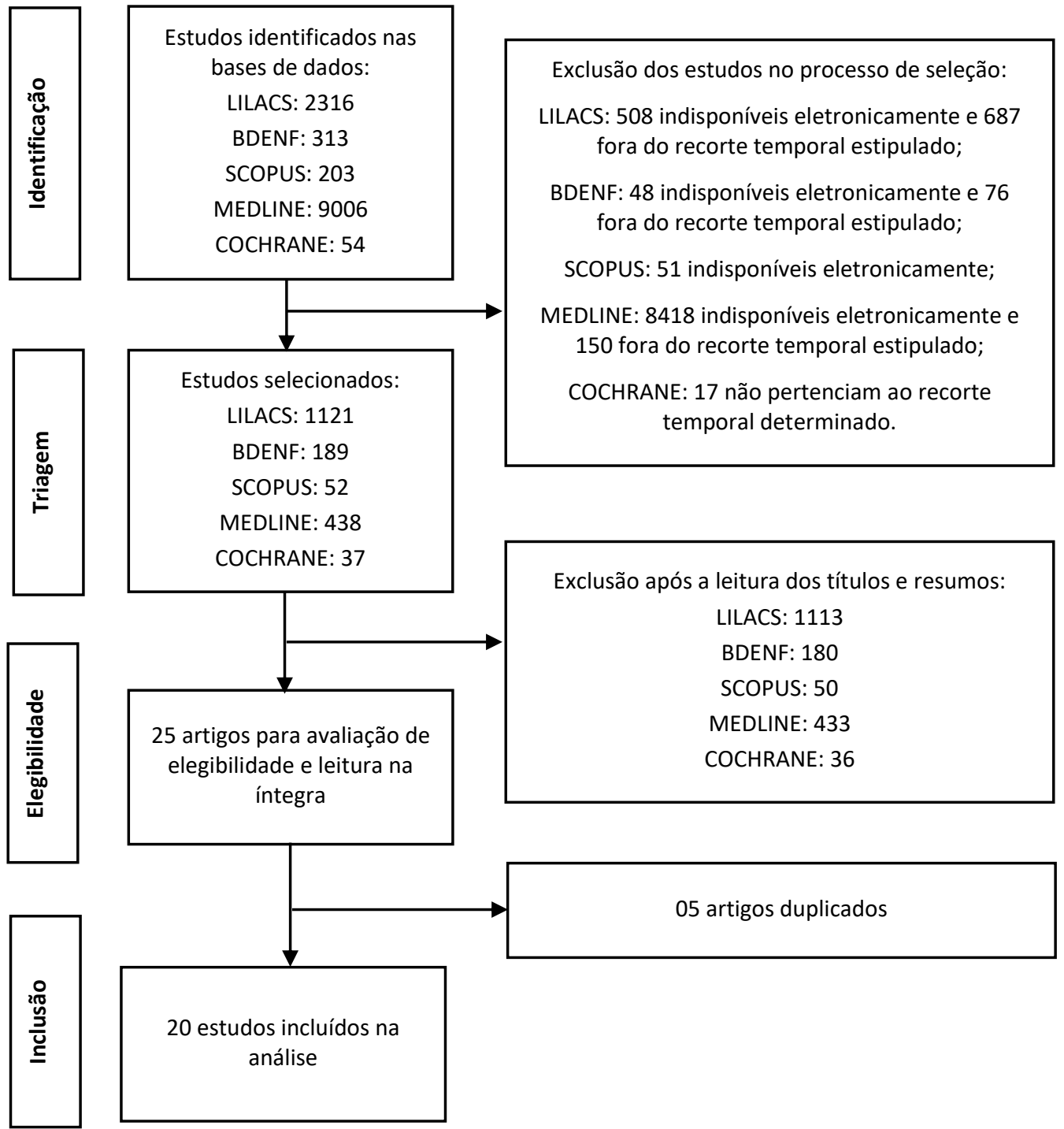

FIGURA 1: Fluxograma do processo de seleção dos estudos, baseado no modelo PRISMA. Sobral, CE, Brasil, 2020.

A seguir, procedeu-se com a leitura aprofundada dos artigos, visando organizar os dados. Para a interpretação dos resultados, os achados foram discutidos a partir da avaliação crítica e utilização de outros estudos que convergem sobre a questão norteadora estabelecida.

Os estudos foram analisados e os dados interpretados de forma organizada e sintetizada por meio de um quadro contendo as seguintes informações: ano, país, desenho metodológico, nível de evidência e periódico. Optou-se também em apresentar as intervenções de enfermagem encontradas em forma de quadro e analisados de acordo com as oito competências para a prática de promoção da saúde definidas na Conferência de Galway ${ }^{10,11}$.

\section{RESULtAdDOS}

Foram localizadas 13 produções, a caracterização dos artigos selecionados apresentada na Figura 2. 


\begin{tabular}{|c|c|c|c|c|c|}
\hline Estudos & Periódico & $\begin{array}{c}\text { Ano de } \\
\text { publicação }\end{array}$ & País de origem & $\begin{array}{c}\text { Desenho } \\
\text { metodológico }\end{array}$ & $\begin{array}{l}\text { Nível de } \\
\text { evidência }\end{array}$ \\
\hline Torres et al. ${ }^{12}$ & $\begin{array}{l}\text { Revista Gaúcha de } \\
\text { Enfermagem }\end{array}$ & 2017 & Brasil & Descritivo & VI \\
\hline $\begin{array}{c}\text { Hafele e } \\
\text { Siqueira }^{13}\end{array}$ & $\begin{array}{l}\text { Revista Brasileira de } \\
\text { Atividade Física e } \\
\text { Saúde }\end{array}$ & 2016 & Brasil & Transversal & IV \\
\hline Dias et al. ${ }^{14}$ & $\begin{array}{l}\text { Journal Health Science } \\
\text { Institute }\end{array}$ & 2016 & Brasil & $\begin{array}{l}\text { Descritivo- } \\
\text { exploratório }\end{array}$ & VI \\
\hline $\begin{array}{l}\text { Mendes,Pontes } \\
\text { e Farias }{ }^{15}\end{array}$ & $\begin{array}{l}\text { Revista Kairós } \\
\text { Gerontologia }\end{array}$ & 2015 & Brasil & $\begin{array}{l}\text { Pesquisa- } \\
\text { participante }\end{array}$ & VI \\
\hline Moura et al. ${ }^{16}$ & $\begin{array}{l}\text { Revista Eletrônica de } \\
\text { Enfermagem }\end{array}$ & 2014 & Brasil & $\begin{array}{l}\text { Estudo de casos } \\
\text { múltiplos }\end{array}$ & VI \\
\hline Silva et al. ${ }^{17}$ & Revista Rene & 2014 & Brasil & Transversal & IV \\
\hline $\begin{array}{c}\text { Pedroni, Rosa, } \\
\text { Almeida e } \\
\text { Guedes. }{ }^{18} \\
\end{array}$ & RECOM & 2013 & Brasil & Transversal & IV \\
\hline $\begin{array}{c}\text { Mendes, Silva } \\
\text { e Ferreira }{ }^{19}\end{array}$ & $\begin{array}{c}\text { Estação científica } \\
\text { (UNIFAP) }\end{array}$ & 2018 & Brasil & Transversal & IV \\
\hline Oliveira et al. ${ }^{20}$ & $\begin{array}{c}\text { Revista de } \\
\text { Enfermagem UFSM }\end{array}$ & 2017 & Brasil & $\begin{array}{l}\text { Descritivo- } \\
\text { exploratório }\end{array}$ & VI \\
\hline $\begin{array}{c}\text { Santos. } \\
\text { Florêncio, } \\
\text { Oliveira e } \\
\text { Moreira }^{21} \\
\end{array}$ & Revista RENE & 2012 & Brasil & $\begin{array}{l}\text { Descritivo- } \\
\text { exploratório }\end{array}$ & VI \\
\hline Oliveira et al. ${ }^{22}$ & $\begin{array}{c}\text { Enfermería } \\
\text { Universitaria ENEO- } \\
\text { UNAM }\end{array}$ & 2012 & México & Descritivo & VI \\
\hline $\begin{array}{l}\text { Resende et } \\
\text { al. }^{23}\end{array}$ & $\begin{array}{c}\text { Revista de } \\
\text { Enfermagem UFPE }\end{array}$ & 2017 & Brasil & Descritivo & VI \\
\hline Sousa et al. ${ }^{24}$ & $\begin{array}{c}\text { Revista de } \\
\text { Enfermagem UFPE }\end{array}$ & 2018 & Brasil & Descritivo & VI \\
\hline $\begin{array}{l}\text { Colósima, Silva, } \\
\text { Toma e Pierin } 25\end{array}$ & $\begin{array}{l}\text { Revista da Escola de } \\
\text { Enfermagem USP }\end{array}$ & 2012 & Brasil & $\begin{array}{l}\text { Ensaio clínico } \\
\text { randomizado }\end{array}$ & II \\
\hline Adler et al. ${ }^{26}$ & BMJ Open & 2019 & Ghana & Coorte & IV \\
\hline Dean et al. ${ }^{27}$ & Family Practice & 2014 & Inglaterra & $\begin{array}{l}\text { Ensaio clínico } \\
\text { randomizado }\end{array}$ & II \\
\hline Zabler et al. ${ }^{28}$ & $\begin{array}{c}\text { Contemporary Clinical } \\
\text { Trials }\end{array}$ & 2018 & Estados Unidos & $\begin{array}{l}\text { Ensaio clínico } \\
\text { randomizado }\end{array}$ & II \\
\hline Enç e Alkan ${ }^{29}$ & Turk Kardiyol Dern Ars & 2017 & Turquia & $\begin{array}{l}\text { Ensaio clínico } \\
\text { randomizado }\end{array}$ & II \\
\hline Hebert et al. $.9^{30}$ & $\begin{array}{l}\text { Journal of General } \\
\text { Internal Medicine }\end{array}$ & 2011 & Estados Unidos & $\begin{array}{l}\text { Ensaio clínico } \\
\text { randomizado }\end{array}$ & II \\
\hline $\begin{array}{l}\text { Guevara, Parra } \\
\text { e Rojas }{ }^{31}\end{array}$ & BMC Nursing & 2019 & Colômbia & $\begin{array}{l}\text { Ensaio Clínico } \\
\text { Randomizado }\end{array}$ & II \\
\hline
\end{tabular}

FIGURA 2 - Quadro Sinóptico dos artigos selecionados para esta revisão. Sobral, CE, Brasil, 2020.

Observou-se prevalência das produções nacionais, que totalizaram 13 artigos (65\%), dentre os quais cinco (25\%) eram oriundos da região Nordeste ${ }^{15,17,21,23,24}$; quatro (20\%) da região Centro-Oeste ${ }^{13,18-20}$, três (15\%) do Sudeste ${ }^{14,16,25}$; e uma (5\%) do Norte ${ }^{12}$.

Observou-se que 7 (35\%) eram de origem internacional, destacando-se os Estados Unidos ${ }^{28,30}$ (10\%); seguido da Inglaterra ${ }^{27}$, México ${ }^{22}$, Gana ${ }^{26}$, Turquia ${ }^{29}$ e Colômbia ${ }^{31}$, com um artigo (5\%) cada.

No que tange ao ano de publicação, destacaram-se os anos de 2017 com quatro artigos ${ }^{12,20,23,29}$ (20\%), seguido dos anos $2012^{21,22,25}, 2014^{16,17,27}$ e $2018^{19,24,28}$, com o quantitativo de três artigos cada (15\%).

Predominaram estudos do tipo descritivo-exploratórios, com sete (35\%), seguido do ensaio clínico randomizado ${ }^{25,27-31}$, com seis (30\%) publicações; quatro (20\%) transversais ${ }^{13,17-19}$; um (5\%) estudo de casos múltiplos ${ }^{16}$, estudo de coorte ${ }^{26}$ e pesquisa-participante ${ }^{15}$, respectivamente. Segundo a classificação dos níveis de evidência dos estudos, nove (45\%) foram classificados em nível VI ${ }^{12,14-16,20-24}$; seis (30\%) nível II ${ }^{25,27-31}$ e cinco (25\%) nível IV ${ }^{13,17-19,26}$. 
A Figura 3 apresenta classificação dos artigos quanto aos domínios de competência e intervenções de enfermagem.

\begin{tabular}{|c|c|}
\hline Domínios de Competência & Intervenções de Enfermagem \\
\hline Catalisar Mudanças & $\begin{array}{l}\text {-Empoderamento do paciente }{ }^{13-15,19-22,24,26-29,31} \\
\text {-Educação em saúde }{ }^{12-16,18-23,25-28,31} \\
\text {-Promoção do autocuidado }{ }^{13-16,18-20,22-24,26,27,30} \\
\text {-Vínculo entre enfermeiro e paciente }{ }^{12,14-16,18,21,24,26} \\
\text {-Desenvolver atividades para promoção de hábitos } \\
\text { saudáveis }{ }^{13-16,18-24,26,27,30,31} \\
\text {-Suporte Emocional12,15-17,19,20,25 } \\
\text {-Identificar barreiras para o tratamento } \\
\text {-Motivação para abandonar vícios } 22,29,31\end{array}$ \\
\hline Liderança & -Comunicação estabelecida ${ }^{12-16,20,21,24,31}$ \\
\hline Avaliação das necessidades & $\begin{array}{l}\text {-Avaliação da qualidade de vida 13,15,17,19,26,27: } \\
\text {-Avaliação da saúde mental }{ }^{12,17,19,22,27} \\
\text {-Avaliação das necessidades da família'16,19,21,22: } \\
\text {-Avaliação do estilo de vida } 13-15,18,20-22,26-28,31\end{array}$ \\
\hline Planejamento & $\begin{array}{l}\text {-Definição de estratégias para promoção da saúde }{ }^{12-16,19-22,26-} \\
\text { 28,31 } \\
\text {-Sistematização da assistência de enfermagem } 16,19,22,23,18,31 \\
\text {-Planejar medidas de enfrentamento }\end{array}$ \\
\hline Implementação & $\begin{array}{l}\text {-Acompanhamento por telefone }{ }^{24} \\
\text { - Realização de visitas domiciliares }{ }^{14,16,19-22,30} \\
\text { - Acompanhamento sistemático das condições de saúde do } \\
\text { paciente } 24-26,30 \\
\text {-Atividades educativas em grupo }{ }^{12,14,18,20,21,25-27} \\
\text { - Adesão à terapêutica farmacológica }{ }^{14,15,17,18,20-22,24,27,28} \\
\text { Suporte social12,14-16,19,20,26 } \\
\text { - Promover estratégias de acesso aos serviços de } \\
\text { saúde }^{12,14,15,28,31} \\
\text {-Promoção da atividade física13,14,16,18-20,22,26 }\end{array}$ \\
\hline Avaliação do impacto & $\begin{array}{l}\text {-Avaliação sistemática da resposta do paciente ao } \\
\text { tratamento/intervenção } 13,15,16,18-20,22,24-31\end{array}$ \\
\hline Defesa & -Possibilitar melhoria do bem estar ${ }^{12,14-16,19,20,26}$ \\
\hline Parcerias & $\begin{array}{l}\text {-Fortalecer Parceria entre enfermeiro, família e paciente }{ }^{12,16-} \\
\text { 19,21,22,24,26-28,31 } \\
\text {-Equipe multidisciplinar }{ }^{13-16,21,26} \\
\text { - Operações conjuntas de atenção primária e serviços sociais } \\
\text { municipais }{ }^{21}\end{array}$ \\
\hline
\end{tabular}

FIGURA 3 - Distribuição dos artigos segundo os domínios de competência e intervenções de enfermagem de acordo com a Conferência de Galway. Sobral, CE, Brasil, 2020.

Verificou-se que $100 \%$ das produções trataram de intervenções que abrangia o domínio de catalisar mudanças, $75 \%$ a avaliação do impacto ${ }^{13,15,16,18-20,22,24-31}$ e 65\% de planejamento ${ }^{12-16,19-22,26-28,31}$.

\section{DISCUSSÃo}

A construção de uma assistência de enfermagem voltada para práticas de promoção da saúde e prevenção de agravos é fundamental para responder às necessidades psíquicas, físicas, socioeconômicas e culturais da pessoa idosa, que deve ser visto pelos profissionais de saúde de forma integral e não fragmentado pelas suas enfermidades ${ }^{32}$.

Alguns estudos desta revisão apresentaram importantes intervenções de educação em saúde ${ }^{12-16,18-23,25-28,31}$ e empoderamento 13-15,19-22,24,26-29,31, ambas pertencentes ao domínio "catalisar mudanças" caracterizado por permitir a transformação por meio da capacitação individual e coletiva no sentido de melhorar a saúde 5 . 
O profissional de enfermagem promove o empoderamento do idoso por meio das ações de educação em saúde que permitem a identificação de barreiras no tratamento e dificuldades de adesão terapêutica e possibilita mudanças no comportamento e adoção de novos hábitos de vida ${ }^{33}$.

Estudo realizado em Unidade Básica de Saúde em Teresina, Piauí, identificou que ações de educação em saúde com vistas ao empoderamento dos pacientes são potentes ferramentas de prevenção de agravos a partir da relação direta mantida entre enfermeiros e idosos, que permitiu a oferta de informações sobre a HAS e orientações acerca de medidas de controle, resultando em uma redução de agravos e complicações decorrentes da doença ${ }^{34}$.

A promoção da saúde está diretamente atrelada ao empoderamento do paciente frente o seu processo de saúde/doença, na medida que possibilita um maior controle sobre decisões e ações relacionados à saúde. Assim, percebe-se a eficácia da educação em saúde quando esta gera na pessoa idosa um protagonismo no enfrentamento de suas vulnerabilidades e na tomada de decisões que influenciam diretamente em um envelhecimento saudável ${ }^{7}$.

A promoção do autocuidado por meio do vínculo entre enfermeiro e paciente também foi apresentada por alguns autores como intervenção capaz de catalisar mudanças em idosos com hipertensão ${ }^{20,23}$. Promover saúde por meio das ações de autocuidado pode revelar um comportamento que busca as potencialidades, entende as limitações, valoriza a saúde e o bem-estar e encontra maneiras criativas de cuidar de $\mathrm{si}^{35}$.

Dessa maneira, a consulta de enfermagem apresenta-se como uma tecnologia fundamental de promoção do autocuidado por meio da sistematização de enfermagem capaz de identificar necessidades reais do paciente, desenvolver um planejamento da assistência e implementar intervenções que potencializam o cuidado, estimulando a pessoa idosa a participar do cuidado com práticas de recuperação da saúde e prevenção de agravos ${ }^{6}$.

Estudo realizado em Minas Gerais, na Estratégia Saúde da Família de um município do interior que objetivou avaliar as ações da Atenção Primária realizadas para melhorar a adesão e o controle da HAS entre idosos, observou que as intervenções e atividades desenvolvidas propiciaram mudanças em relação aos fatores de risco a partir da relação do enfermeiro com os participantes da pesquisa, como a adoção de estilo de vida saudável e adesão à medicação ${ }^{16}$.

A terceira competência de promoção da saúde trata-se da avaliação de necessidades. Para essa finalidade, alguns estudos apontaram como estratégia a avaliação da qualidade de vida dos idosos ${ }^{13,15,17,19,26,27}$. Assim sendo, é necessário reforçar a importância de avaliar a qualidade de vida dessa população com a possibilidade de implementar ações interventivas de promoção da saúde e de prevenção, de forma individual e coletiva, auxiliando na modificação de comportamentos de riscos e na adesão de hábitos de vida mais saudáveis ${ }^{36}$.

Outras intervenções que se inserem na competência da avaliação de necessidades, é avaliação da saúde mental ${ }^{12,19,22,27}$ e do estilo de vida ${ }^{13-15,18,20-22,26-28,32}$. Estudos sugerem que esses indivíduos ao serem expostos a situações difíceis, apresentariam dificuldades no manejo da doença, comprometendo a adesão ao tratamento e, consequentemente, sua qualidade de vida ${ }^{5,17}$. Desse modo, o enfermeiro surge como profissional que identifica fatores de risco no contexto psicológico e do estilo de vida, traçando estratégias eficazes de promoção da saúde que provoquem mudanças comportamentais e ações de enfrentamento à estressores ${ }^{37}$.

A fim de potencializar o cuidado a pessoa idosa com HAS, é necessário que o enfermeiro assuma na sua prática a competência do Planejamento. Dentre os estudos selecionados, a Sistematização de Enfermagem ${ }^{16,19,22,23,28,31}$ e a adoção de medidas de enfrentamento ${ }^{13-16,18-22,25,27,28}$ destacaram-se. Nesse ínterim, a utilização do Processo de Enfermagem (PE) possibilita a aplicação da ampla estrutura teórica de enfermagem à prática clínica, tornando possível a atenção individualizada, ordenada e sistematizada, além de auxiliar o paciente no enfrentamento da enfermidade ${ }^{37}$.

No contexto do cuidado do idoso com hipertensão, o PE deve ser compreendido como um modelo tecnológico complexo que possibilita ofertar conforto, bem-estar físico e mental ao paciente. Em estudo que buscou utilizar o PE em 45 usuários da Estratégia Saúde da Família e cadastrados no HIPERDIA, evidenciou-se que o levantamento de diagnósticos de enfermagem estava associado a uma melhor análise das respostas à hipertensão, bem como favoreceu um cuidado mais integral e centrado no indivíduo, não apenas em sua doença ${ }^{16}$.

A implementação, quinta competência, é a realização efetiva e eficiente de estratégias culturalmente sensíveis e éticas para garantir o maior grau possível de melhorias na saúde, incluindo a gestão de recursos humanos e materiais ${ }^{5}$. Identificou-se nesse domínio intervenções como abordagem grupal12,14,18,20,21,25,27, visita domiciliar ${ }^{14,16,19-22,30}$, acompanhamento sistemático do paciente ${ }^{24,26,30}$, adesão terapêutica farmacológica ${ }^{14,15,17,18,20-22,24,27,28}$ e suporte social ${ }^{12,14-16,19,20,26}$.

A educação grupal, como abordagem em saúde, possibilita troca de conhecimentos, favorece a educação em saúde e a identificação de fatores de riscos dos sujeitos envolvidos. No contexto da gerontologia, constitui importante ferramenta de promoção da saúde, utilizada no incentivo à prática de atividade física, alimentação saudável, melhoria do padrão cognitivo e autoeficácia do cuidado para reduzir os fatores de risco modificáveis ${ }^{36}$. Grupos constituídos por 
pessoas idosas utilizam estratégias de empoderamento e autonomia, com mudanças positivas para todos os atores envolvidos no processo educativo.

Já a visita domiciliar, surge como um meio de cuidado e promoção da saúde, de busca ativa e identificação da demanda que muitas vezes não chega até a unidade de saúde, além de propiciar o diagnóstico local e subsidiar o planejamento de ações a partir da realidade, de mediação entre as famílias e as equipes de saúde ${ }^{38}$. Estudo relata a experiência de uma intervenção educativa realizada com dez idosos por meio de visitas domiciliares, que proporcionaram estabelecimento de vínculo e relação de confiança entre pesquisadores e participantes, além de ter favorecido uma melhor compreensão e esclarecimento de dúvidas dos participantes e familiares ${ }^{21}$.

No que concerne a adesão terapêutica farmacológica, foi evidenciado como essencial no tratamento de pessoas idosas com HAS, pois necessitam de um esclarecimento de qualidade acerca dos benefícios das medicações juntamente de um acompanhamento regular, em particular aqueles pacientes resistentes ao regime medicamentoso ${ }^{27,28}$. Ademais, muitos pacientes apresentam dificuldade cognitiva em lembrar de tomar a medicação, o que prejudica na continuidade efetiva do tratamento.

O suporte social é uma variável relevante na prevenção de doenças, na promoção da saúde, na adesão terapêutica e no processo de recuperação ${ }^{25}$. Um programa educacional, citado entre os artigos, tinha o objetivo de auxiliar idosos que enfrentam doenças crônicas sem auxílio de cuidadores ou familiares a buscar maior suporte social e, com isso, ajudar na resolução de problemas e na diminuição do estresse. Além disso, o programa realizava escuta terapêutica entre os pacientes através da construção de um grupo de apoio ${ }^{15}$.

A sexta competência, avaliação do impacto, objetiva determinar o alcance, a eficácia e as repercussões das políticas e dos programas de promoção da saúde. Isso inclui a utilização de avaliação de qualidade e métodos de investigação para apoiar programas de melhorias e sustentabilidade ${ }^{5}$. A maioria dos $\operatorname{artigos}^{13,15,16,18-19,22,24-31}$ selecionados do estudo realizaram uma avaliação sistemática da resposta do paciente ao tratamento favorecendo o planejamento de intervenções e em sua implementação.

A defesa do paciente e das comunidades, outro domínio de competências encontrado entre os artigos analisados ${ }^{12,14,16,19,21,22}$, visa à melhoria da saúde e do bem-estar, quando favorece aspectos importantes de qualidade de vida e promoção da saúde de pessoas idosas hipertensas ${ }^{5}$. O profissional deve sentir-se corresponsável pelo paciente, principalmente quando se trata de populações vulneráveis, como o caso de idosos e pacientes com hipertensão.

Com base na oitava competência, o trabalho cooperativo entre disciplinas, setores e parceiros pode melhorar o impacto e a sustentabilidade de programas e políticas de promoção da saúde ${ }^{5}$, foi evidenciado em alguns estudos a parceria com a família $12,16,19,21,22,24,26,31$, com a equipe interdisciplinar ${ }^{13-15}$, havendo encaminhamento do paciente para outros setores ou serviços ${ }^{21}$. Nesse sentido, o enfermeiro, enquanto coordenador do cuidado, deve buscar meios de integrar a equipe multiprofissional para atenderas necessidades sociais e de saúde que a velhice demanda, por meio de ações preventivas, curativas e reabilitadoras que contribuam na melhoria da qualidade de vida da pessoa idosa ${ }^{33}$.

De acordo com os dados apresentados neste estudo, são várias as competências que os enfermeiros podem desenvolver para a promoção da saúde de idosos com hipertensão arterial, dentre estas: realização de intervenções tendo em vista a geração de mudanças, avaliação de necessidades dos indivíduos, orientações quanto aspectos importantes da qualidade de vida e hábitos saudáveis, bem como melhorar o impacto de práticas e políticas de promoção da saúde e estabelecer parcerias interprofissionais e intersetoriais.

Mediante o exposto, compreende-se a importância de uma formação profissional de enfermagem capacitada e direcionada às práticas de promoção da saúde, a fim de potencializar uma assistência integral ao idosos, priorizando a identificação e atendimento às suas necessidades.

Identificar as competências de enfermagem na promoção de saúde de idosos com hipertensão à luz do Consenso de Galway possibilita ao enfermeiro maior clareza e visibilidade do seu fazer, resultando no aumento da qualidade da assistência para um público com vulnerabilidades clínicas e sociais. Além disso, amplia a capacidade de análise das necessidades do idoso e de intervenção no contexto de saúde/doença dessa população.

\section{Limitações do estudo}

Como limitação desta revisão, ressalta-se a quantidade reduzida de bases selecionadas e a delimitação da língua dos estudos durante a busca. Dessa maneira, recomenda-se que para construção de novas pesquisas sobre a temática, sejam utilizadas um maior número de bases a fim de ampliar a busca e consequentemente a identificação de outras práticas promotoras da saúde realizadas por enfermeiros. 


\section{CONCLUSÃO}

Foram analisados 20 artigos sobre as competências de enfermagem de promoção da saúde para idosos hipertensos. Os dados apresentados neste estudo apontam várias competências que os enfermeiros desenvolvem, tais como: avaliação integral do paciente, educar em saúde, desenvolver estratégias de empoderamento, prevenir agravos, avaliar estilo de vida, intervir em fatores de risco modificáveis e promover parcerias entre paciente, familiares e profissionais, planejamento e avaliação. Os domínios de competência mais evidenciados nas publicações foram: Catalisar mudança, Avaliação do impacto e Planejamento.

Salienta-se que os resultados deste estudo poderão subsidiar as condutas dos enfermeiros para a prática da promoção da saúde em pessoas idosas com hipertensão, bem como estimular tais profissionais à adoção das estratégias aqui discutidas.

\section{REFERÊNCIAS}

1. Malta DC, Bernal RTI, Lima MG, Araújo SSC, Silva MMA, Freitas MIF, et al. Noncommunicable diseases and the use of health services: analysis of the National Health Survey in Brazil. Rev. Saúde Publica. [Internet]. 2017 [cited 2020 May 15]; 51:Supl 1:4s. DOI: https://doi.org/10.1590/S1518-8787.2017051000090.

2. Schenker M, Costa DH. Advances and challenges of health care of the elderly population with chronic diseases in Primary Health Care. Ciênc. saúde coletiva. [Internet]. 2019 [cited 2020 May 15]; 24(4):1369-80. DOI: https://doi.org/10.1590/141381232018244.01222019.

3. Nascimento MGG, Nadaleti NP, Vilela SC, Terra FS, Silva AS, Resck ZMR. Nursing work process in the promotion of mental health: reflective analysis. Revista de Enfermagem do Centro Oeste Mineiro. [Internet]. 2017 [cited 2020 May 15]; 7:2097. DOI: https://doi.org/10.19175/recom.v7i0.2097.

4. Carvalho LV, Melo GM, Aquino PS, Castro RCMB, Cardoso MVLML, Pagliuca LMF. Assistive technologies for the blind: key competences for health promotion under the Galway Consensus. Rev. Rene. [Internet]. 2017 [cited 2020 May 15]; 18(3):412-9. DOI: https://doi.org/10.15253/2175-6783.2017000300018.

5. Allegrante JP, Barry MM, Airhihenbuwa CO, Auld ME, Collins JL, Magnusson MCLG, et al. Domains of core competency, standards and quality assurance for building global capacity in health promotion: the Galway Consensus Conference Statement. Health Education \& Behavior. [Internet]. 2009 [cited 2020 May 15]; Vol. 36(3):476-82. DOI: https://doi.org/10.1177/1090198109333950.

6. Souza MT, Silva MD, Carvalho R. Integrative review: what is it? how to do it? Einstein [Internet]. 2010 [cited 2020 May 15]; 8(1):102-6. Available from: https://www.scielo.br/pdf/eins/v8n1/1679-4508-eins-8-1-0102.pdf.

7. Carvalho KM, Silva CR, Figueiredo ML, Nogueira LT, Andrade EM. Educational interventions for the health promotion of the elderly: integrative review. Acta Paul Enferm. [Internet]. 2018 [cited 2020 May 15]; 31(4):446-54. DOI: http://dx.doi.org/10.1590/1982-0194201800062.

8. Ministério da Saúde (Br). Secretaria de Vigilância em Saúde. Secretaria de Atenção à Saúde. Departamento de Análise de Situação e Saúde. Política Nacional de Promoção da Saúde. Brasília (DF): Ministério da Saúde; 2010.

9. Galvão TF, Pansani TSA, Harrad D. Main items to report Systematic reviews and Meta-analyzes: The PRISMA recommendation. Epidemiol. Serv. Saúde. [Internet]. 2015; 24(2):335-42. DOI: https://doi.org/10.5123/S1679-49742015000200017.

10. Carvalho VL de, Oliveira ALC de, Alves IKS, Silva RL, Silva CB. Health promotion competencies of undergraduate health professionals. Rev. enferm. UFPE on line. [Internet]. 2017 [cited 2020 May 15]; 11(Supl. 8):3269-78. DOI: https://doi.org/10.5205/reuol.11135-99435-1-ED.1108sup201711.

11. Cestari VRF, Florêncio RS, Moreira TMM Pessoa VLMP, Barbosa IV, Lima FET, et al. Nursing competencies in promoting the health of individuals with chronic diseases. Rev. bras. enferm. [Internet]. 2016 [cited 2020 May 15]; 69(6):1195-1203. DOI: http://dx.doi.org/10.1590/0034-7167-2016-0312.

12. Torres GMC, Figueiredo IDT, Cândido JAB, Pinto AAG, Morais APP, Araújo MFM, et al. Therapeutic communication in the interaction between health workers and hypertensive patients in the family health strategy. Rev. gaúcha enferm. [Internet] 2017 [cited 2020 May 15]; 38(4):e2016-0066. DOI: http://dx.doi.org/10.1590/1983-1447.2017.04.2016-0066.

13. Häfele V, Siqueira FV. Physical activity counseling and change of behavior in Basic Health Units. Rev Bras Ativ Fís Saúde. [Internet]. 2016 [cited 2020 May 15];21(6):581-92. DOI: https://doi.org/10.12820/rbafs.v.21n6p581-592.

14. Dias EGD, Almeida FG, Caires HLD, Santos TAS, Jorge AS, Mishima SM. Evaluation of a Family Health Strategy about the promotion of adherence to treatment and control of hypertension under the optics of the elderly. J Health Sci Inst. [Internet]. 2016 [cited 2020 May 15]; 34(2):88-92. Available from: https://unip.br/presencial/comunicacao/publicacoes/ics/edicoes/2016/02_abr-jun/V34_n2_2016_p88a92.pdf.

15. Mendes, AJB, Ponte, KMA, Farias, MS. Nursing care for membership habits of elderly living healthy with Health Promotion of the Theory of base. Revista Kairós-Gerontologia. [Internet]. 2015 [cited 2020 May 15]; 18(4):269-87. DOI: https://doi.org/10.23925/2176-901X.2015v18i4p269-287.

16. Moura DJMM, Freitas MC, Guedes MVC, Lopes MVO, Menezes LCG, Barros AA. Systematization of nursing care based on CIPE ${ }^{\circledR}$ and the theory of adaptation in hypertensives. Rev. Eletr. Enf. [Internet.] 2014 [cited 2020 May 15]; 16(4):710-9. DOI: http://dx.doi.org/10.5216/ree.v16i4.22945. 
17. Silva PCS, Monteiro LA, Graciano ADS, Terra FS, Veiga EV. Assessment of depression in elderly with systemic hypertension. Rev. Rene. [Internet]. 2014 [cited 2020 May 15]; 15(1):151-7. DOI: https://doi.org/10.15253/2175-6783.2014000100019.

18. Pedroni GAM, Rosa JA, ALmeida MEF, Guedes HM. Nursing care for the elderly with hypertension. R. Enferm. Cent. O. Min. [Internet].2013 [cited 2020 May 15]; 3(2):662-9. DOI: https://doi.org/10.19175/recom.v0i0.379.

19. Mendes FA, Silva MP, Ferreira CRS. Nursing diagnostics in patients with arterial hypertension in primary care. Estação Científica (UNIFAP). [Internet]. 2018 [cited 2020 May 15]; 8(1):91-101. DOI: http://dx.doi.org/10.18468/estcien.2018v8n1.p91-101.

20. Oliveira MSNO, Almeida GBSA, Chagas DNP, Salazar PR, Ferreira LV. Self-care of elderly diagnosed with arterial hypert Ension and/or diabetes mellitus. Rev. Enferm. UFSM. [Internet]. 2017 [cited 2020 May 15]; 7(3):490-503. DOI: https://doi.org/10.5902/2179769226344.

21. Santos JC, Florêncio RS, Oliveira CJ, Moreira TMM. Elderly adherence to hypertension treatment and nursing interventions. Rev Rene. [Internet]. 2012 [cited 2020 May 15]; 13(2):343-53. Available from: http://periodicos.ufc.br/rene/article/view/3924.

22. Salcedo-Álvarez RA, González-Caamaño BC, Jiménez MA, Nava RV, Cortés ODA, Eugenio RMB. Self-Care for hypertension in elderly outpatients, an approach to the NANDA-NOC-NIC taxonomy. Enfermería Universitária [Internet]. 2012 [cited 2020 Dec 15]; 9(3):25-43. Available from: http://www.scielo.org.mx/scielo.php?script=sci_arttext\&pid=S1665$70632012000300004 \& \operatorname{lng}=\mathrm{es}$.

23. Resende AKM, Lira JAC, Prudêncio FA, Sousa LS, Brito JFP, Ribeiro, JF, et al. Difficulties of elderly people in accession to the treatment of blood hypertension. Rev. enferm. UFPE on line. [Internet]. 2018 [cited 2020 May 15]; 12(10):2546-54. DOI: https://doi.org/10.5205/1981-8963-v12i10a236078p2546-2554-2018.

24. Sousa RC, Lucena ALR, Nascimento WS, Ferreira TMC, Lima CL, Ferreira JDL, el al. Particularities of hypertensive elderly people to medicinal treatment adherence. J. Nurs. UFPE on line. [Internet]. 2018 [cited 2020 May 15]; 12(1):216-23. Available from: https://periodicos.ufpe.br/revistas/revistaenfermagem/article/viewFile/23296/26002.

25. Colósimo FC, Silva SSBE da, Toma G de A, Pierin AMG. Nursing actions increases the control of hypertensive patients and reduces white-coat effect. Rev. esc. enferm. USP [Internet]. 2012 [cited 2020 Dec 15]; 46(spe):10-5. DOI: https://doi.org/10.1590/S0080-62342012000700002.

26. Adler AJ, Laar A, Prieto-Merino D, Der RMM, Mangortey, Dirks R, et al. Can a nurse-led community-based model of hypertension care improve hypertension control in Ghana? Results from the Com HIP cohort study. BMJ Open. [Internet]. 2019 [cited 2020 May 15]; 9:e026799. DOI: https://doi.org/10.1136/bmjopen-2018-026799.

27. Sally CD, Sally MK, Teck KK, Sarah RK, Pippa O. Avaliação de uma clínica especializada em hipertensão dirigida por uma enfermeira com apoio de consultor em duas práticas gerais do centro da cidade: ensaio clínico randomizado. Clínica Familiar. [Internet]. 2014 [cited 2020 May 17]; 31(2):172-9. DOI: https://doi.org/10.1093/fampra/cmt07.4.

28. Zabler B, Tsai PY, Fendrich M, Cho Y, Taani MH, Schiffman R. Effect of a nurse case management intervention for hypertension self-management in low-income African Americans. Contemp Clin Trials. [Internet]. 2018 [cited 2020 May 15]; 71:199-204. DOI: https://doi.org/10.1016/j.cct.2018.06.011.

29. Enç N, Öz Alkan H. Nursing care in elderly patients with cardiovascular disease. Turk Kardiyol Dern Ars. [Internet]. 2017 [cited 2020 May 15]; 45(Suppl 5):120-3. DOI: https://doi.org/10.5543/tkda.2017.92147.

30. Hebert PL, Sisk JE, Tuzzio L, Casabianca JM, Pogue VA, Wang JJ, et al. Nurse-led disease management for hypertension control in a diverse urban community: a randomized trial. J. Gen. Intern. Med. [Internet]. 2012 [cited 2020 May 15];27(6):630-9. DOI: https://doi.org/10.1007/s11606-011-1924-1.

31. Castro APR, Vidal ECF, Saraiva ARB, Arnaldo SM, Borges AMM, Almeida MI. Promoting health among the elderly: actions in primary health care. Rev. bras. geriatr. gerontol. [Internet]. 2018 [cited 2020 May 15]; 21(2):158-67.DOI: http://dx.doi.org/10.1590/1981-22562018021.170133.

32. Tavares MFL, Rocha RM, Bittar CML, Petersen CB, Andrade M. Health promotion in professional education: challenges in Health and the need to achieve in other sectors. Ciênc. saúde Colet. [Internet]. 2016 [cited 2020 May 15]; 21(6):1799-808. DOI: https://doi.org/10.1590/1413-81232015216.07622016.

33. Penha MM, Silva TMM, Coelho LS, Azevedo AP, Costa AWS, Silva RA, et al. The perception of hypertensive elderly people to nursing care in a family health strategy. Rev. UNINGÁ. [Internet]. 2018 [cited 2020 May 15]; 55(1):146-54. Available from: http://revista.uninga.br/index.php/uninga/article/view/56.

34. Esteves M, Vendramini SHF, Santos M de LSG, Brandão VZ, Soler ZASG, Lourenção LG. Quality of life of hypertensive and diabetic elderly in an outpatient clinic. Medicina [Internet]. 2017 [cited 2020 May 15]; 50(1):18-2. DOI: https://doi.org/10.11606/issn.2176-7262.v50i1p18-28.

35. Santos MG, Bitencourt JVOV, Silva TG, Frizon G, Quinto AS. Stages of the nursing process: a narrative review. Enferm. Foco. [Internet]. 2017 [cited 2020 May 15]; 8(4):49-53. DOI: https://doi.org/10.21675/2357-707X.2017.v8.n4.1032.

36. Silva GM, Ness O, Guanaes-Lorenzi C. Continuing Education in Mental Health: Critical Moments to Analyze Group Process. Paidéia [Internet]. 2018 [cited 2020 May 15]; 28:e2834. DOI: http://dx.doi.org/10.1590/1982-4327e2834.

37. Santos GS, Cunha ICKO. Home visit to elderly: features and associated factors. Revista de Enfermagem do Centro Oeste Mineiro. [Internet]. 2017 [cited 2020 May 15]; 7:e1271. DOI: https://doi.org/10.19175/recom.v7i0.1271.

38. Sousa VLP, Moreira ACA, Oliveira FES, Magalhães Júnior JWC. Nursing skills in the health promotion of elderly people with mental disorder. Rev enferm UERJ. [Internet]. 2019 [cited 2020 May 15]; 27:e43242. DOI: http://dx.doi.org/10.12957/reuerj.2019.43242. 